\title{
Some new cyclic admissibility type with uni-dimensional and multidimensional fixed point theorems and its applica- tions
}

\author{
Chirasak Mongkolkeha ${ }^{a}$, Wutiphol Sintunavarat ${ }^{\text {b,* }}$ \\ a Department of Mathematics, Statistics and Computer Sciences, Faculty of Liberal Arts and Science, Kasetsart University, \\ Kamphaeng-Saen Campus, Nakhonpathom 73140, Thailand. \\ ${ }^{b}$ Department of Mathematics and Statistics, Faculty of Science and Technology, Thammasat University Rangsit Center, Pathumthani \\ 12121, Thailand.
}

Communicated by Y. J. Cho

\begin{abstract}
In this paper, we introduce the concept of a cyclic $(\alpha, \beta)$-admissible mapping type $S$ and the notion of an $(\alpha, \beta)-(\psi, \varphi)$ contraction type $S$. We also establish fixed point results for such contractions along with the cyclic $(\alpha, \beta)$-admissibility type $S$ in complete b-metric spaces and provide some examples for supporting our result. Applying our new results, we obtain fixed point results for cyclic mappings and multidimensional fixed point results. As application, the existence of a solution of the nonlinear integral equation is discussed.
\end{abstract}

Keywords: $\alpha$-admissible mappings, cyclic $(\alpha, \beta)$-admissible mappings, generalized weak contraction mappings, multidimensional fixed points, nonlinear integral equations.

2010 MSC: 47H09, 47H10, 54H25.

(C)2018 All rights reserved.

\section{Introduction}

The well-known Banach's contraction principle has been generalized in many ways over the years $([5,7,9,13,15])$. One of the most interesting is the extension of Banach's contractions to case of weakly contractions which was first given by Alber et al. [3] in the framework of Hilbert spaces. Afterward, Rhoades [16] claimed that the Alber et al.'s fixed point result in [3] is also valid in complete metric spaces. In 2008, Dutta and Choudhury [12] extended the weak contractions by using the concept of an altering distance function, i.e., a function that alters distance between two points in a metric space, which was first introduced by Khan et al. [14]. They also established some fixed point results for such contractions in complete metric spaces.

In 2012, Samet et al. [18] introduced the famous concept of a very useful tool for proving the existence

\footnotetext{
*Corresponding author

Email addresses: faascsm@ku.ac.th (Chirasak Mongkolkeha), wutiphol@mathstat.sci.tu.ac.th (Wutiphol Sintunavarat)

doi: $10.22436 /$ jnsa.011.09.04
}

Received: 2017-07-07 Revised: 2018-03-22 Accepted: 2018-04-15 
theorems of fixed points which is called $\alpha$-admissibility. Also, they established various fixed point theorems for new nonlinear mapping satisfying some contractions in complete metric spaces. Later, in 2014, Alizadeh et al. [4] introduced the similar notion with $\alpha$-admissibility of Samet et al. [18], which is called cyclic $(\alpha, \beta)$-admissibility. They also obtained fixed point results for nonlinear mappings concerning such admissibility in the setting of complete metric spaces. Very recently, Sintunavarat [19] defined the notion of a new admissibility type which is called $\alpha$-admissibility type $S$. That is the motivation to write this work. He also showed that the class of $\alpha$-admissible mappings and the class of $\alpha$-admissible mappings type $S$ are independent and proved fixed point results for nonlinear mapping including this new class in b-metric spaces.

From above mentioned, we combine the concept of a cyclic $(\alpha, \beta)$-admissibility with the idea in [19] for defining the new type of a cyclical-admissibility called cyclic $(\alpha, \beta)$-admissibility type $S$. We also give some examples to show that the class of cyclic $(\alpha, \beta)$-admissible mappings and cyclic $(\alpha, \beta)$-admissible mappings type $S$ are independent. Moreover, we introduce the new contraction namely $(\alpha, \beta)-(\psi, \varphi)-$ contraction type $S$ and give the sufficient condition to guarantee the existence of fixed point results for nonlinear mapping satisfying such contractions in b-metric spaces. We offer an illustrative example to indicate the validity of the hypotheses and degree of utility of our results. As an application of our results, fixed point results for cyclic mappings, multidimensional fixed point results and the existence result of a solution for the nonlinear integral equation are given.

\section{Preliminaries}

In this section, we give some notations and basic knowledges. Throughout this paper, we denote by $\mathbb{N}$ and $\mathbb{R}$ the sets of positive integers and real numbers, respectively.

\subsection{History of various kinds of contractions}

The first well-known contractions were first introduced by Banach in his thesis [6] as follows.

Definition 2.1 ([6]). A self-mapping $T$ on a metric space $(X, d)$ is said to be contraction if there is $k \in[0,1)$ satisfying

for all $x, y \in X$.

$$
d(T x, T y) \leqslant k d(x, y)
$$

In 1997, Rhoades [16] suggested the following contraction which was first introduced by Alber et al. [3] in the setting of inner product spaces.

Definition 2.2 ([16]). A self-mapping $T$ on a metric space $(X, d)$ is said to be weak contraction if

$$
d(T x, T y) \leqslant d(x, y)-\varphi(d(x, y))
$$

for all $x, y \in X$, where $\varphi:[0, \infty) \rightarrow[0, \infty)$ is a continuous and nondecreasing function with $\varphi(0)=0$ and $\varphi(t)>0$ for all $t>0$.

Remark 2.3. If we take $\phi(t)=(1-k) t$ for all $t \geqslant 0$, where $0 \leqslant k<1$, then the condition (2.2) becomes (2.1).

Rhoades [16] also established the following fixed point result for nonlinear mappings satisfying the weak contractive condition.

Theorem 2.4 ([16]). Let $(\mathrm{X}, \mathrm{d})$ be a complete metric space and $\mathrm{T}: \mathrm{X} \rightarrow \mathrm{X}$ satisfies the weak contractive condition (2.2). Then $\mathrm{T}$ has a unique fixed point.

In 1984, Khan et al. [14] introduced the concept of an altering distance function as follows.

Definition 2.5 ([14]). A function $\psi:[0, \infty) \rightarrow[0, \infty)$ is called an altering distance function if the following properties are satisfied:

1. $\psi$ is continuous and monotone nondecreasing; 
2. $\psi(t)=0$ if and only if $t=0$.

Note that if $\psi$ is an altering distance function, then it commutes with $\max$, i.e., $\psi\left(\max \left\{s_{1}, s_{2}, \ldots, s_{N}\right\}\right)=$ $\max \left\{\psi\left(s_{1}\right), \psi\left(s_{2}\right), \ldots, \psi\left(s_{N}\right)\right\}$ for all $s_{1}, s_{2}, \ldots, s_{N} \in[0, \infty)$.

Here, we give some examples of an altering distance function.

Example 2.6. Let $\varphi_{i}:[0, \infty) \rightarrow[0, \infty), i \in\{1,2, \ldots, 5\}$ be defined by

$\left(\varphi_{1}\right) \varphi_{1}(t)=k t$ for all $t \in[0, \infty)$, where $k>0$;

$\left(\varphi_{2}\right) \varphi_{2}(t)=t^{k}$ for all $t \in[0, \infty)$, where $k>0$;

$\left(\varphi_{3}\right) \varphi_{3}(\mathrm{t})= \begin{cases}\mathrm{t}^{2}, & \mathrm{t} \in[0,1] \\ 1+\sqrt{\mathrm{t}-1}, & \mathrm{t} \in(1, \infty) ;\end{cases}$

$\left(\varphi_{4}\right) \varphi_{4}(\mathrm{t})=\mathrm{a}^{\mathrm{t}}-1$ for all $\mathrm{t} \in[0, \infty)$, where $\mathrm{a}>0$ and $\mathrm{a} \neq 1$;

$\left(\varphi_{5}\right) \varphi_{5}(t)=\log (k t+1)$ for all $t \in[0, \infty)$, where $k>0$.

Then $\varphi_{i}$ is an altering distance function for each $i \in\{1,2, \ldots, 5\}$.

In 2008, Dutta and Choudhury [12] extended the concept of a weak contraction by using the concept of two altering distance functions and proved the fixed point results for such contractions as follows.

Theorem 2.7. Let $(\mathrm{X}, \mathrm{d})$ be a complete metric space and $\mathrm{T}: \mathrm{X} \rightarrow \mathrm{X}$ be a mapping satisfying the inequality

$$
\psi(d(T x, T y)) \leqslant \psi(d(x, y))-\varphi(d(x, y))
$$

for all $x, y \in X$, where $\psi, \varphi:[0, \infty) \rightarrow[0, \infty)$ are two altering distance functions. Then $T$ has a unique fixed point.

Afterward, Dorić [11] (see also [1]) replaced the continuity of $\varphi$ by "lower semi-continuous" and proved the following results.

Theorem 2.8 ([11]). Let $(\mathrm{X}, \mathrm{d})$ be a complete metric space and $\mathrm{T}: \mathrm{X} \rightarrow \mathrm{X}$ be a mapping satisfying the inequality

$$
\psi(d(T x, T y)) \leqslant \psi(N(x, y))-\varphi(N(x, y))
$$

for all $x, y \in X$, where $N(x, y)$ is given by

$$
N(x, y):=\max \left\{d(x, y), d(x, T x), d(y, T y), \frac{d(x, T y)+d(y, T x)}{2}\right\},
$$

$\psi:[0, \infty) \rightarrow[0, \infty)$ is an altering distance function and $\varphi:[0, \infty) \rightarrow[0, \infty)$ is a lower semi-continuous function with $\varphi(t)=0$ if and only if $\mathrm{t}=0$. Then $\mathrm{T}$ has a unique fixed point.

\subsection{History of various types of admissibility}

In 2012, Samet et al. [18] introduced the concept of an $\alpha$-admissibility which is a very useful tool for proving the existence of fixed points as follows.

Definition 2.9 ([18]). Let $X$ be a nonempty set and $\alpha: X \times X \rightarrow[0, \infty)$ be a mapping. A self mapping $\mathrm{T}: \mathrm{X} \rightarrow \mathrm{X}$ is said to be $\alpha$-admissible if the following condition holds:

$$
x, y \in X, \alpha(x, y) \geqslant 1 \Longrightarrow \alpha(T x, T y) \geqslant 1
$$

Example 2.10 ([18]). Let $X=[0, \infty)$ and define $T: X \rightarrow X$ and $\alpha: X \times X \rightarrow[0, \infty)$ by

$$
\mathrm{T} x=\sqrt{x} \text { for all } x \in X
$$

and

$$
\alpha(x, y)= \begin{cases}e^{x-y}, & x \geqslant y \\ 0, & x<y\end{cases}
$$

Then, $\mathrm{T}$ is $\alpha$-admissible. 
In 2014, Alizadeh et al. [4] introduced the notion of a cyclic $(\alpha, \beta)$-admissible mapping as follows.

Definition 2.11 ([4]). Let $X$ be a nonempty set, $T: X \rightarrow X$ be a mapping, and $\alpha, \beta: X \rightarrow[0, \infty)$ be two functions. We say that $T$ is a cyclic $(\alpha, \beta)$-admissible mapping if the following conditions hold:

1. $\alpha(x) \geqslant 1$ for some $x \in X$ implies $\beta(T x) \geqslant 1$;

2. $\beta(x) \geqslant 1$ for some $x \in X$ implies $\alpha(T x) \geqslant 1$.

Example 2.12 ([4]). Let $X=\mathbb{R}$ and $T: X \rightarrow X$ be defined by $T(x)=-\left(x+x^{3}\right)$ for all $x \in X$ and let $\alpha, \beta: X \rightarrow[0, \infty)$ be defined by $\alpha(x)=e^{x}$ for all $x \in X$ and $\beta(x)=e^{-x}$ for all $x \in X$. Then $T$ is a cyclic $(\alpha, \beta)$-admissible mapping.

Recently, Sintunavarat [19] defined the notion of a new admissibility type which called $\alpha$-admissible mapping type $S$.

Definition 2.13 ([19]). Let $X$ be a nonempty set, $s$ be a given real number such that $s \geqslant 1$ and $\alpha: X \times X \rightarrow$ $[0, \infty)$ be a given mapping. A mapping $T: X \rightarrow X$ is said to be an $\alpha$-admissible mapping type $S$ if the following condition holds:

$$
x, y \in X \text { with } \alpha(x, y) \geqslant s \Longrightarrow \alpha(f x, f y) \geqslant s .
$$

Example 2.14 ([19]). Let $X=\mathbb{R}, s=2$ and mappings $\alpha: X \times X \rightarrow[0, \infty)$ and $T: X \rightarrow X$ be defined by

$$
\alpha(x, y)=\left\{\begin{array}{ll}
x^{2}+y^{2}, & x, y \in[3,4], \\
\min \{1,|x-y|\}, & \text { otherwise, }
\end{array} \text { and } T x= \begin{cases}3+\tanh (2 x+1), & x \in[3,4], \\
\frac{x}{2}, & x \in[0,3) \cup(4, \infty) .\end{cases}\right.
$$

Then we can see that $\mathrm{T}$ is an $\alpha$-admissible mapping type $S$. Moreover, $T$ is not an $\alpha$-admissible mapping.

\section{3. b-metric spaces}

In 1993, Czerwik [10] introduced the concept of a b-metric space which is a generalization of the metric space as follows.

Definition 2.15 ([10]). Let $X$ be a nonempty set and $s \geqslant 1$ be a given real number. Suppose that the mapping $d: X \times X \rightarrow[0, \infty)$ satisfies the following conditions:

$\left(B M_{1}\right) d(x, y)=0$ if and only if $x=y$;

$\left(B M_{2}\right) d(x, y)=d(y, x)$ for all $x, y \in X$;

$\left(B_{3}\right) d(x, y) \leqslant s[d(x, z)+d(z, y)]$ for all $x, y, z, \in X$.

Then $(X, d)$ is called a b-metric space with the coefficient $s \geqslant 1$.

It is obvious that the class of b-metric spaces is effectively larger than that of metric spaces since any metric space is a $b$-metric space with $s=1$. The following examples show that, in general, a $b$-metric space need not necessarily be a metric space.

Example 2.16. Let $X=\mathbb{R}$ and the mapping $d: X \times X \rightarrow[0, \infty)$ be defined by

$$
d(x, y)=(x-y)^{2} \quad \text { for all } x, y \in X .
$$

Then $(X, d)$ is a b-metric space with the coefficient $s=2$.

Example 2.17 ([19]). Let $(X, d)$ be a metric space and the mapping $\sigma_{d}: X \times X \rightarrow[0, \infty)$ be defined by

$$
\sigma_{d}(x, y)=[d(x, y)]^{p} \quad \text { for all } x, y \in X,
$$

where $p>1$ is a fixed real number. Then $\left(X, \sigma_{d}\right)$ is a b-metric space with the coefficient $s=2^{p-1}$. 
Next, we give the concepts of b-convergence, b-Cauchy sequence, b-continuity and b-completeness in b-metric spaces.

Definition $2.18([8])$. Let $(X, d)$ be a b-metric space. Then a sequence $\left\{x_{n}\right\}$ in $X$ is called:

(a) b-convergent if there exists $x \in X$ such that $d\left(x_{n}, x\right) \rightarrow 0$ as $n \rightarrow \infty$. In this case, we write $\lim _{n \rightarrow \infty} x_{n}=x$;

(b) b-Cauchy if $d\left(x_{n}, x_{m}\right) \rightarrow 0$ as $n, m \rightarrow \infty$.

Each b-convergent sequence in b-metric spaces has a unique limit and it is also a b-Cauchy sequence. Moreover, in general, a b-metric is not continuous. So we need the following simple lemma about the $\mathrm{b}$-convergent sequences in the proof of our main result.

Lemma 2.19 ([2]). Let $(\mathrm{X}, \mathrm{d})$ be a b-metric space with coefficient $\mathrm{s} \geqslant 1$ and let $\left\{x_{\mathrm{n}}\right\}$ and $\left\{\mathrm{y}_{\mathrm{n}}\right\}$ be $\mathrm{b}$-convergent to points $x, y \in X$, respectively. Then we have

$$
\frac{1}{s^{2}} d(x, y) \leqslant \liminf _{n \rightarrow \infty} d\left(x_{n}, y_{n}\right) \leqslant \limsup _{n \rightarrow \infty} d\left(x_{n}, y_{n}\right) \leqslant s^{2} d(x, y)
$$

In particular, if $x=y$, then we have $\lim _{n \rightarrow \infty} \mathrm{d}\left(x_{n}, y_{n}\right)=0$. Moreover, for each $z \in X$, we have

$$
\frac{1}{s} d(x, z) \leqslant \liminf _{n \rightarrow \infty} d\left(x_{n}, z\right) \leqslant \limsup _{n \rightarrow \infty} d\left(x_{n}, z\right) \leqslant s d(x, z)
$$

Definition 2.20 ([8]). Let $\left(X, d_{X}\right)$ and $\left(Y, d_{Y}\right)$ be two b-metric spaces.

1. The space $\left(X, d_{X}\right)$ is $b$-complete if every $b$-Cauchy sequence in $X$ b-converges.

2. A function $f: X \rightarrow Y$ is $b$-continuous at a point $x \in X$ if it is b-sequentially continuous at $x$, that is, whenever $\left\{x_{n}\right\}$ is b-convergent to $x,\left\{f x_{n}\right\}$ is b-convergent to $f x$.

Definition 2.21 ([8]). Let $Y$ be a nonempty subset of a b-metric space $(X, d)$. The closure of $Y$ is denoted by $\bar{Y}$ and it is the set of limits of all convergent sequences of points in $Y$, i.e.,

$$
\bar{Y}:=\left\{x \in X: \text { there exists a sequence }\left\{x_{n}\right\} \text { in } Y \text { such that } \lim _{n \rightarrow \infty} x_{n}=x\right\} \text {. }
$$

Definition 2.22 ([8]). A subset $Y$ of a $b$-metric space $(X, d)$ is closed if and only if for each sequence $\left\{x_{n}\right\}$ in $Y$ which $b$-converges to an element $x \in X$, we have $x \in Y$ (i.e. $Y=\bar{Y}$ ).

\subsection{Multidimensional}

Let $n$ be a positive integer, $X$ a nonempty set and $X^{n}$ denotes the product space $\overbrace{X \times X \times \cdots \times X}^{n \text {-terms }}$. We let $\{A, B\}$ be a partition of $\Lambda_{n}=\{1,2, \ldots, n\}$, that is, $A \cup B=\Lambda_{n}$ and $A \cap B=\varnothing$. Then we denote:

$$
\Omega_{A, B}=\left\{\sigma: \Lambda_{n} \rightarrow \Lambda_{n}: \sigma(A) \subseteq A \text { and } \sigma(B) \subseteq B\right\}
$$

and

$$
\Omega_{A, B}^{\prime}=\left\{\sigma: \Lambda_{n} \rightarrow \Lambda_{n}: \sigma(A) \subseteq B \text { and } \sigma(B) \subseteq A\right\} .
$$

Henceforth, let $\sigma_{1}, \sigma_{2}, \ldots, \sigma_{n}$ be $n$ mappings from $\Lambda_{n}$ into itself and let $\Upsilon$ be the (n)-tuple $\left(\sigma_{1}, \sigma_{2}, \ldots, \sigma_{n}\right)$.

Definition 2.23. ([17]) Let $X$ be a nonempty set. A point $\left(x_{1}, x_{2}, \ldots, x_{n}\right) \in X^{n}$ is called a $\Upsilon$-coincidence point of the mapping $F: X^{n} \rightarrow X$ and $g: X \rightarrow X$ if for all $i \in\{1,2, \ldots n\}$,

$$
F\left(x_{\sigma_{i}(1)}, x_{\sigma_{i}(2)}, \ldots, x_{\sigma_{i}(n)}\right)=g x_{i} .
$$

If $g$ is identity mapping on $X$, then $\left(x_{1}, x_{2}, \ldots, x_{n}\right) \in X^{n}$ is called ar-fixed point of $F$. 


\section{Uni-dimensional fixed point theorems for $(\alpha, \beta)-(\psi, \varphi)$-contractions type $S$}

In this section, we introduce the concept of a cyclic $(\alpha, \beta)$-admissible mapping type $S$ and prove the fixed point theorems for such mappings.

Now, we present the new type of a cyclic $(\alpha, \beta)$-admissible mapping so called cyclic $(\alpha, \beta)$-admissible mapping type $S$ as follows:

Definition 3.1. Let $X$ be a nonempty set, $s$ be a given real number such that $s \geqslant 1, T: X \rightarrow X$ be a mapping, and $\alpha, \beta: X \rightarrow[0, \infty)$ be two mappings. We say that $T$ is a cyclic $(\alpha, \beta)$-admissible mapping type $S$ if

1. $\alpha(x) \geqslant s$ for some $x \in X$ implies $\beta(T x) \geqslant s$;

2. $\beta(x) \geqslant s$ for some $x \in X$ implies $\alpha(T x) \geqslant s$.

Next, we give some examples to show that the class of cyclic $(\alpha, \beta)$-admissible mappings and the class of cyclic $(\alpha, \beta)$-admissible mappings type $S$ are different.

Example 3.2. Let $X=[0, \infty)$ with $s=2$ and $T: X \rightarrow X$ be defined by

$$
\mathrm{Tx}= \begin{cases}3+|\sin x|, & x \in[3,4] \\ \frac{1}{x+1}, & x \in[0,3) \cup(4, \infty) .\end{cases}
$$

Define two mappings $\alpha, \beta: X \rightarrow[0, \infty)$ by

$$
\alpha(x)=\left\{\begin{array}{ll}
x^{2}, & x \in[3,4], \\
1, & \text { otherwise, }
\end{array} \quad \text { and } \quad \beta(x)= \begin{cases}2 x, & x \in[3,4] \\
\frac{1}{x+1}, & \text { otherwise }\end{cases}\right.
$$

Here we claim that $T$ is a cyclic $(\alpha, \beta)$-admissible mapping type $S$. If $\alpha(x) \geqslant 2$, then we have $x \in[3,4]$ and so $T x=3+|\sin x| \in[3,4]$. Consequently $\beta(T x) \geqslant 2$. If $\beta(x) \geqslant 2$, then we have $x \in[3,4]$ and so $T x=$ $3+|\sin x| \in[3,4]$. Consequently $\alpha(T x) \geqslant 2$. Then $T$ is a cyclic $(\alpha, \beta)$-admissible mapping type $S$. Further, $\mathrm{T}$ is not a cyclic $(\alpha, \beta)$-admissible mapping. Indeed, if $x=1$ then $\alpha(x)=1$, but $\beta(T x)=\beta(1 / 2)=2 / 3<1$.

Example 3.3. Let $X=\mathbb{R}$ with $s=2$ and $T: X \rightarrow X$ be defined by

$$
\mathrm{T} x= \begin{cases}-\frac{x}{2^{\prime}} & x \in(-\infty,-2] \cup\{0\} \cup[2, \infty), \\ -\left(x+x^{3}\right), & \text { otherwise. }\end{cases}
$$

Define two mappings $\alpha, \beta: X \rightarrow[0, \infty)$ by

$$
\alpha(x)=\left\{\begin{array}{ll}
2, & x=0, \\
x+\cosh (3 x+2), & x \in[2, \infty), \\
e^{x}, & \text { otherwise, }
\end{array} \quad \text { and } \quad \beta(x)= \begin{cases}2+\ln |x+1|, & x \in(-\infty,-2], \\
e^{-x}, & \text { otherwise. }\end{cases}\right.
$$

Here we claim that $T$ is a cyclic $(\alpha, \beta)$-admissible mapping. If $\alpha(x) \geqslant 1$, then we must have $x \in[0, \infty)$.

Case I: $x \in[2, \infty)$, then $T x \in(-\infty,-1]$. Clearly, in case $T x \in(-\infty,-2]$, we get $\beta(T x) \geqslant 2>1$. On the other hand, $T x \in(-2,-1]$, then $\beta(T x)=e^{-T x} \geqslant 1$.

Case II: $x \in[0,2)$, then $\beta(T x)=e^{-T x} \geqslant 1$.

If $\beta(x) \geqslant 1$, then we must have $x \in(-\infty, 0]$.

Case I: $x \in(-\infty,-2]$, then $\mathrm{T} x \in[1, \infty)$. Clearly, in case $\mathrm{T} x \in[2, \infty)$, we get $\alpha(\mathrm{T} x) \geqslant 2>1$. Otherwise, $T x \in[1,2)$, then $\alpha(T x)=e^{T x}>1$. 
Case II: $x \in(-2,0]$, then $\alpha(T x) \geqslant 1$.

Therefore $T$ is cyclic $(\alpha, \beta)$-admissible mapping. Moreover, $T$ is not cyclic $(\alpha, \beta)$-admissible mapping type $S$ with $s=2$. Indeed, if $x=0$ then $\alpha(x)=2=s$, but $\beta(T x)=\beta(0)=e^{0}=1<2$.

Throughout this work, unless otherwise stated, $F(T)$ stands for the set of all fixed points of self mapping $T$ on a nonempty set $X$, that is,

$$
\mathrm{F}(\mathrm{T}):=\{x \in \mathrm{X} \mid \mathrm{T} x=\mathrm{x}\}
$$

Unless otherwise specified, we denote by $\Psi$ and $\Phi$ the following sets

$$
\Psi:=\{\psi:[0, \infty) \rightarrow[0, \infty): \psi \text { is an altering distance function }\}
$$

and

$$
\Phi:=\{\varphi:[0, \infty) \rightarrow[0, \infty): \varphi \text { is lower semi-continuous with } \varphi(t)=0 \text { if and only if } t=0\} .
$$

Definition 3.4. Let $(X, d)$ be a b-metric space with the coefficient $s \geqslant 1$ and $\alpha, \beta: X \rightarrow[0, \infty)$ be two given mappings. A mapping $T: X \rightarrow X$ is said to be an $(\alpha, \beta)-(\psi, \varphi)$-contraction mapping type $S$ if and only if

$$
x, y \in X, \quad \alpha(x) \beta(y) \geqslant s \Rightarrow \psi\left(s^{3} d(T x, T y) \leqslant \psi(d(x, y))-\varphi(d(x, y)),\right.
$$

where $\psi \in \Psi$ and $\varphi \in \Phi$.

Theorem 3.5. Let $(\mathrm{X}, \mathrm{d})$ be a complete $\mathrm{b}$-metric space with the coefficient $\mathrm{s} \geqslant 1$, and $\mathrm{T}: \mathrm{X} \rightarrow \mathrm{X}$ be an $(\alpha, \beta)$ $(\psi, \varphi)$-contraction mapping type $\mathrm{S}$ and the following conditions hold:

(a) $\mathrm{T}$ is a cyclic $(\alpha, \beta)$-admissible mapping type $S$;

(b) there exists $x_{0} \in X$ such that $\alpha\left(x_{0}\right) \geqslant s$ and $\beta\left(x_{0}\right) \geqslant s$;

(c) $\mathrm{T}$ is continuous, or

(c') if $\left\{x_{n}\right\}$ is a sequence in $X$ and it converges to $x \in X$ and $\beta\left(x_{n}\right) \geqslant s$ for all $n \in \mathbb{N}$ then $\beta(x) \geqslant s$.

Then $\mathrm{T}$ has a fixed point in $\mathrm{X}$. Furthermore, if $\alpha(x) \beta(\mathrm{y}) \geqslant s$ for all $\mathrm{x}, \mathrm{y} \in \mathrm{F}(\mathrm{T})$, then the fixed point of $\mathrm{T}$ is unique.

Proof. Let $x_{0} \in X$ be a point in the assumption (b) and then $\alpha\left(x_{0}\right) \geqslant s$ and $\beta\left(x_{0}\right) \geqslant s$. Define the sequence $\left\{x_{n}\right\}$ by $x_{n}=T^{n} x_{0}=T x_{n-1}$ for all $n \in \mathbb{N}$. From the fact that $T$ is a cyclic $(\alpha, \beta)$-admissible mapping type $S$ and $\alpha\left(x_{0}\right) \geqslant s$, we get $\beta\left(x_{1}\right)=\beta\left(T x_{0}\right) \geqslant s$ which implies that $\alpha\left(x_{2}\right)=\alpha\left(T x_{1}\right) \geqslant s$. Continuing this method, we get $\alpha\left(x_{2 n}\right) \geqslant s$ and $\beta\left(x_{2 n-1}\right) \geqslant s$ for all $n \in \mathbb{N}$. By similar argument, we can see that $\alpha\left(x_{2 n-1}\right) \geqslant s$ and $\beta\left(x_{2 n}\right) \geqslant s$ for all $n \in \mathbb{N}$. This yields that $\alpha\left(x_{n}\right) \geqslant s$ and $\beta\left(x_{n}\right) \geqslant s$ for all $n \in \mathbb{N} \cup\{0\}$ and hence $\alpha\left(x_{n-1}\right) \beta\left(x_{n}\right) \geqslant s$ for all $n \in \mathbb{N}$. By the $(\alpha, \beta)-(\psi, \varphi)$-contractive type $S$ condition of $T$, we have

$$
\psi\left(s^{3} d\left(x_{n}, x_{n+1}\right) \leqslant \psi\left(d\left(x_{n-1}, x_{n}\right)\right)-\varphi\left(d\left(x_{n-1}, x_{n}\right)\right)\right.
$$

for all $n \in \mathbb{N}$. Since $\psi$ is nondecreasing and $s \geqslant 1$, we get $d\left(x_{n}, x_{n+1}\right) \leqslant s^{3} d\left(x_{n}, x_{n+1}\right) \leqslant d\left(x_{n-1}, x_{n}\right)$. This mean that the sequence $\left\{d\left(x_{n}, x_{n+1}\right)\right\}$ is nonincreasing and hence there exists some non-negative real number $r$ such that

$$
\lim _{n \rightarrow \infty} d\left(x_{n}, x_{n+1}\right)=r .
$$

Taking the limit supremum as $n \rightarrow \infty$ in (3.1), by using (3.2) and the property of $\psi$ and $\varphi$, we have

$$
\psi\left(s^{3} r\right) \leqslant \psi(r)-\varphi(r) \leqslant \psi(r) .
$$

This which implies that $\varphi(r)=0$ and thus $r=0$. Hence,

$$
\lim _{n \rightarrow \infty} d\left(x_{n}, x_{n+1}\right)=0 .
$$


Next, we prove that $\left\{x_{n}\right\}$ is a b-Cauchy sequence in $X$. Suppose the contrary, then there exists $\epsilon>0$ for which we can find subsequences $\left\{x_{m(k)}\right\}$ and $\left\{x_{n(k)}\right\}$ of $\left\{x_{n}\right\}$ with $n(k)>m(k) \geqslant k$ such that

$$
\mathrm{d}\left(\mathrm{x}_{\mathrm{m}(\mathrm{k})}, \mathrm{x}_{\mathrm{n}(\mathrm{k})}\right) \geqslant \epsilon
$$

and $n(k)$ is the smallest number such that (3.4) holds. That is

$$
\mathrm{d}\left(\mathrm{x}_{\mathrm{m}(\mathrm{k})}, \mathrm{x}_{\mathrm{n}(\mathrm{k})-1}\right)<\epsilon
$$

By $\left(\mathrm{BM}_{3}\right),(3.4)$, and (3.5), we get

$$
\epsilon \leqslant d\left(x_{m(k)}, x_{n(k)}\right) \leqslant s\left[d\left(x_{m(k)}, x_{n(k)-1}\right)+d\left(x_{n(k)-1}, x_{n(k)}\right)\right]<s \epsilon+d\left(x_{n(k)-1}, x_{n(k)}\right) .
$$

Taking the limit supremum as $k \rightarrow \infty$ in above inequality, by using (3.3) we get

$$
\epsilon \leqslant \operatorname{limsupd}_{k \rightarrow \infty}\left(x_{m(k)}, x_{n(k)}\right) \leqslant s \epsilon .
$$

Since,

$$
d\left(x_{m(k)}, x_{n(k)}\right) \leqslant s\left[d\left(x_{m(k)}, x_{n(k)+1}\right)+d\left(x_{n(k)+1}, x_{n(k)}\right)\right]
$$

and

$$
d\left(x_{m(k)}, x_{n(k)+1}\right) \leqslant s\left[d\left(x_{m(k)}, x_{n(k)}\right)+d\left(x_{n(k)}, x_{n(k)+1}\right)\right] .
$$

Taking limit supremum as $k \rightarrow \infty$ in (3.7) and (3.8), by using from (3.3) and (3.6), we get

$$
\epsilon \leqslant s\left(\operatorname{limsupd}_{k \rightarrow \infty}\left(x_{\mathfrak{m}(k)}, x_{n(k)+1}\right)\right)
$$

and

$$
\operatorname{limsupd}_{k \rightarrow \infty} d\left(x_{m(k)}, x_{n(k)+1}\right) \leqslant s^{2} \epsilon .
$$

By the inequalities (3.9) and (3.10), we have

$$
\frac{\epsilon}{s} \leqslant \operatorname{limsupd}_{k \rightarrow \infty}\left(x_{m(k)}, x_{n(k)+1}\right) \leqslant s^{2} \epsilon .
$$

Similarly, we can show that

$$
\frac{\epsilon}{s} \leqslant \operatorname{limsupd}_{k \rightarrow \infty}\left(x_{m(k)+1}, x_{n(k)}\right) \leqslant s^{2} \epsilon
$$

Finally, since

$$
\begin{aligned}
d\left(x_{m(k)+1}, x_{n(k)+1}\right) & \leqslant s\left[d\left(x_{m(k)+1}, x_{m(k)}\right)+d\left(x_{m(k)}, x_{n(k)+1}\right)\right] \\
& \leqslant s d\left(x_{m(k)+1}, x_{m(k)}\right)+s^{2}\left[d\left(x_{m(k)}, x_{n(k)}\right)+d\left(x_{n(k)}, x_{n(k)+1}\right)\right],
\end{aligned}
$$

it follows that

$$
\operatorname{limsupd}_{k \rightarrow \infty}\left(x_{m(k)+1}, x_{n(k)+1}\right) \leqslant s^{3} \epsilon .
$$

By $\left(B M_{3}\right)$, we get

$$
\begin{aligned}
d\left(x_{m(k)}, x_{n(k)}\right) & \leqslant s\left[d\left(x_{m(k)}, x_{m(k)+1}\right)+d\left(x_{m(k)+1}, x_{n(k)}\right)\right] \\
& \leqslant s d\left(x_{m(k)}, x_{m(k)+1}\right)+s^{2}\left[d\left(x_{m(k)+1}, x_{n(k)+1}\right)+d\left(x_{n(k)+1}, x_{n(k)}\right)\right] .
\end{aligned}
$$

Taking limit supremum as $k \rightarrow \infty$ in (3.12) and using (3.3) and (3.6), we have,

$$
\frac{\epsilon}{s^{2}} \leqslant \operatorname{limsupd}_{k \rightarrow \infty}\left(x_{m}(k)+1, x_{n(k)+1}\right) .
$$


By inequalities (3.11) and (3.13), we have

$$
\frac{\epsilon}{s^{2}} \leqslant \operatorname{limsupd}_{k \rightarrow \infty}\left(x_{m}(k)+1, x_{n}(k)+1\right) \leqslant s^{3} \epsilon .
$$

Since, $\alpha\left(x_{n_{k}}\right) \beta\left(x_{m_{k}}\right) \geqslant s$ for all $k \in \mathbb{N}$. By the $(\alpha, \beta)-(\psi, \varphi)$-contraction type $S$ of $T$, we have

$$
\psi\left(s^{3} d\left(x_{m(k)+1}, x_{n(k)+1}\right)\right) \leqslant \psi\left(d\left(x_{m(k)}, x_{n(k)}\right)\right)-\varphi\left(d\left(x_{m(k)}, x_{n(k)}\right)\right) .
$$

Letting $k \rightarrow \infty$ in (3.15) and using (3.14), (3.6), and the properties of $\psi$ and $\varphi$, we have

$$
\begin{aligned}
\psi(s \epsilon)=\psi\left(s^{3}\left(\frac{\epsilon}{s^{2}}\right)\right) & \leqslant \psi\left(s^{3} \operatorname{limsupd}_{k \rightarrow \infty}\left(x_{\mathfrak{m}(k)+1}, x_{\mathfrak{n}(k)+1}\right)\right) \\
& \leqslant \psi\left(\operatorname{limsupd}_{k \rightarrow \infty}\left(x_{\mathfrak{m}(k)}, x_{n}(k)\right)\right)-\varphi\left(\liminf _{k \rightarrow \infty}\left(x_{\mathfrak{m}(k)}, x_{n(k)}\right)\right) \leqslant \psi(s \epsilon)-\varphi(\epsilon) .
\end{aligned}
$$

This implies that $\varphi(\epsilon)=0$ and hence $\epsilon=0$, which is a contradiction. Therefore, $\left\{x_{n}\right\}$ is a b-Cauchy sequence. By the completeness of $b$-metric space $X$, there exists $x \in X$ such that

$$
\lim _{n \rightarrow \infty} d\left(x_{n}, x\right)=0
$$

Assume that the assumption (c) holds, then we get

$$
\lim _{n \rightarrow \infty} d\left(T x_{n}, T x\right)=0
$$

Since,

$$
d(x, T x) \leqslant s\left[d\left(x, T x_{n}\right)+d\left(T x_{n}, T x\right)\right]
$$

for all $n \in \mathbb{N} \cup\{0\}$. Taking limit as $n \rightarrow \infty$ in above inequality, we obtain

$$
d(x, T x)=0
$$

and hence $T x=x$. Now, assume that the assumption $\left(c^{\prime}\right)$ holds, then we get $\alpha\left(x_{n}\right) \beta(x) \geqslant s$. Since $T$ is an $(\alpha, \beta)-(\psi, \varphi)$-contraction mapping type $S$, we have

$$
\psi\left(s^{3} d\left(x_{n+1}, T x\right)\right) \leqslant \psi\left(d\left(x_{n}, x\right)\right)-\varphi\left(d\left(x_{n}, x\right)\right)
$$

for all $n \in \mathbb{N}$. Taking the limit supremum as $n \rightarrow \infty$ in (3.16), we get $\psi\left(s^{3} d(x, T x)\right) \leqslant 0$. Hence, $\mathrm{d}(x, T x)=0$, that is $T x=x$.

For proving the uniqueness of fixed point of $T$, suppose that $x$ and $x^{*}$ are two fixed points of $T$ with $x \neq x^{*}$. From the hypothesis, we obtain that $\alpha(x) \beta\left(x^{*}\right) \geqslant s$. Since $T$ is an $(\alpha, \beta)-(\psi, \varphi)$-contraction mapping type $S$, we have

$$
\psi\left(s^{3} d\left(x, x^{*}\right)\right)=\psi\left(s^{3} d\left(T x, T x^{*}\right)\right) \leqslant \psi\left(d\left(x, x^{*}\right)\right)-\varphi\left(d\left(x, x^{*}\right)\right)<\psi\left(d\left(x, x^{*}\right)\right) .
$$

This yields that $s^{3} d\left(x, x^{*}\right)<d\left(x, x^{*}\right)$, which is a contradiction. Hence $x=x^{*}$. This completes the proof.

Example 3.6. Let $X=\mathbb{R}$ with the $b$-metric $d: X \times X \rightarrow[0, \infty)$ given by $d(x, y)=(x-y)^{2}$ for all $x, y \in X$. Then $(X, d)$ is a complete $b$-metric space with the coefficient $s=2$. Define the mappings $T: X \rightarrow X$ and $\alpha, \beta: X \rightarrow[0, \infty)$ by

$$
\mathrm{T} x=-\sinh ^{-1} \frac{x}{4} \text { for all } x \in X, \quad \alpha(x)=\left\{\begin{array}{ll}
2, & \text { if } x \in[0, \infty), \\
0, & \text { otherwise }
\end{array} \quad \text { and } \quad \beta(x)= \begin{cases}2, & \text { if } x \in(-\infty, 0], \\
0 & \text { otherwise }\end{cases}\right.
$$


Also, we define $\psi$ and $\varphi$ by $\psi(t)=k t$ and $\varphi(t)=\frac{k t}{2}$ for all $t \in[0, \infty)$, where $1<k<2$.

Now we will show that $T$ is a cyclic $(\alpha, \beta)$-admissible mapping type $S$. Let $\alpha(x) \geqslant s=2$ for some $x \in X$. Then $x \in[0, \infty)$ and $T x \in(-\infty, 0]$. Thus $\beta(T x) \geqslant 2$. Similarly, if $\beta(x) \geqslant s=2$ for some $x \in X$, then $x \in(-\infty, 0]$ and $T x \in[0, \infty)$ and hence $\alpha(T x) \geqslant 2$. Hence, $T$ is a cyclic $(\alpha, \beta)$-admissible mapping type $S$.

Next, we will prove that $T$ is an $(\alpha, \beta)-(\psi, \varphi)$-contraction mapping type $S$. Suppose that $x, y \in X$ such that $\alpha(x) \beta(y) \geqslant s=2$. Then we get $x \in[0, \infty)$ and $y \in(-\infty, 0]$ and so we have

$$
\begin{aligned}
\psi\left(2^{3} d(T x, T y)\right)=8 k(T x-T y)^{2} & =8 k\left(\sinh ^{-1} \frac{x}{4}-\sinh ^{-1} \frac{y}{4}\right)^{2} \\
& \leqslant 8 k\left(\frac{x}{4}-\frac{y}{4}\right)^{2}=\frac{k}{2}(x-y)^{2} \\
& =k(x-y)^{2}-\frac{k}{2}(x-y)^{2}=\psi(d(x, y))-\varphi(d(x, y)) .
\end{aligned}
$$

Then $T$ is $(\alpha, \beta)-(\psi, \varphi)$-contraction type $S$.

It is easy to see that there is $x_{0} \in X$ such that $\alpha\left(x_{0}\right) \geqslant s$ and $\beta\left(x_{0}\right) \geqslant s$. Moreover, $T$ is continuous. Therefore, all conditions of Theorem 3.5 hold. So we can conclude that $T$ has a fixed point, that is, a point $0 \in X$.

Remark 3.7. By using the same technique in Theorem 3.5, we can replace the condition (c) or $\left(\mathrm{c}^{\prime}\right)$ by the following condition:

- if $\left\{x_{n}\right\}$ is a sequence in $X$ that converges to $x \in X$ and $\alpha\left(x_{n}\right) \geqslant s$ for all $n \in \mathbb{N}$, then $\alpha(x) \geqslant s$.

From Theorem 3.5, we can proof the following corollary and then the details are omitted.

Corollary 3.8. Let $(X, d)$ be a complete b-metric space with constant $s \geqslant 1, \alpha, \beta: X \rightarrow[0, \infty)$ be two given mappings and $\mathrm{T}: \mathrm{X} \rightarrow \mathrm{X}$ be a mapping satisfying the following inequality:

$$
\alpha(x) \beta(y) \psi\left(s^{3} d(T x, T y)\right) \leqslant \psi(d(x, y))-\varphi(d(x, y))
$$

for all $x, y \in X$, where $\psi \in \Psi$ and $\varphi \in \Phi$. Suppose that the following conditions hold:

(a) $T$ is a cyclic $(\alpha, \beta)$-admissible mapping type $S$;

(b) there exists $x_{0} \in X$ such that $\alpha\left(x_{0}\right) \geqslant s$ and $\beta\left(x_{0}\right) \geqslant s$;

(c) $T$ is continuous, or

$\left(c^{\prime}\right)$ if $\left\{x_{n}\right\}$ is a sequence in $X$ that converges to $x \in X$ and $\beta\left(x_{n}\right) \geqslant s$ for all $n$, then $\beta(x) \geqslant s$, or

$\left(c^{\prime \prime}\right)$ if $\left\{x_{n}\right\}$ is a sequence in $X$ that converges to $x \in X$ and $\alpha\left(x_{n}\right) \geqslant s$ for all $n$, then $\alpha(x) \geqslant s$.

Then $T$ has a fixed point in $X$. Furthermore, if $\alpha(x) \beta(y) \geqslant s$ for all $x, y \in F(T)$, then the fixed point of $T$ is unique.

It has been pointed out in some studies that the fixed point results for cyclic mappings can be concluded from Theorem 3.5 under some suitable cyclic $(\alpha, \beta)$-admissible mappings type $S$.

Theorem 3.9. Let $\mathrm{A}$ and $\mathrm{B}$ be two closed subsets of a complete $\mathrm{b}$-metric space $(\mathrm{X}, \mathrm{d})$ with constant $\mathrm{s} \geqslant 1$ such that $\mathrm{A} \cap \mathrm{B} \neq \emptyset$ and $\mathrm{T}: \mathrm{A} \cup \mathrm{B} \rightarrow \mathrm{A} \cup \mathrm{B}$ be a cyclic mapping, i.e., $\mathrm{T}(\mathrm{A}) \subseteq \mathrm{B}$ and $\mathrm{T}(\mathrm{B}) \subseteq \mathrm{A}$, satisfying

$$
\psi\left(s^{3} d(T x, T y)\right) \leqslant \psi(d(x, y))-\varphi(d(x, y))
$$

for all $\mathrm{x} \in \mathrm{A}$ and $\mathrm{y} \in \mathrm{B}$, where $\psi \in \Psi$ and $\varphi \in \Phi$. Then $\mathrm{T}$ has a unique fixed point in $\mathrm{A} \cap \mathrm{B}$.

Proof. Let $\alpha, \beta: X \rightarrow[0, \infty)$ be defined by

$$
\alpha(x)=\left\{\begin{array}{ll}
s, & \text { if } x \in A, \\
0, & \text { otherwise, }
\end{array} \quad \text { and } \quad \beta(x)= \begin{cases}s, & \text { if } x \in B \\
0, & \text { otherwise }\end{cases}\right.
$$


Now we will show that $T$ is an $(\alpha, \beta)-(\psi, \varphi)$-contraction mapping type $S$. Assume that $x, y \in X$ with $\alpha(x) \beta(y) \geqslant s$. Then we have $x \in A$ and $y \in B$. By the inequality (3.17), we have

$$
\psi\left(s^{3} d(T x, T y)\right) \leqslant \psi(d(x, y))-\varphi(d(x, y)) .
$$

So $T$ is an $(\alpha, \beta)-(\psi, \varphi)$-contraction mapping type $S$.

Next, we will show that $T$ is a cyclic $(\alpha, \beta)$-admissible mapping type $S$.

- If $\alpha(x) \geqslant s$ for some $x \in X$, then $x \in A$ and $T x \in B$ and thus $\beta(T x) \geqslant s$.

- If $\beta(x) \geqslant s$ for some $x \in X$, then $x \in B$ and $T x \in A$ and hence $\alpha(T x) \geqslant s$.

Therefore, $T$ is a cyclic $(\alpha, \beta)$-admissible mapping type $S$.

It follows from the definitions of $\alpha$ and $\beta$ and the fact that $A \cap B \neq \emptyset$ that there is $x_{0} \in A \cap B$ such that $\alpha\left(x_{0}\right) \geqslant s$ and $\beta\left(x_{0}\right) \geqslant s$.

Finally, we will show that the condition ( $\left.c^{\prime}\right)$ in Theorem 3.5 holds. Assume that $\left\{x_{n}\right\}$ is a sequence in $X$ such that $\beta\left(x_{n}\right) \geqslant s$ for all $n \in \mathbb{N}$ and $x_{n} \rightarrow x_{\star}$ as $n \rightarrow \infty$. Then $x_{n} \in B$ for all $n \in \mathbb{N}$. Since B is a closed subset of $X$, we obtain $x_{\star} \in B$ and so $\beta\left(x_{\star}\right) \geqslant s$.

Therefore, all conditions in Theorem 3.5 hold and thus $T$ has a fixed point in $A \cup B$. Assume that $z \in A \cup B$ is a fixed point of $T$. If $z \in A$, then $z=T z \in B$. In case $z \in B$, we get $z=T z \in A$. Therefore, $z \in A \cap B$. Moreover, the fixed point of $T$ is unique since $\alpha(x) \beta(y)=s^{2} \geqslant s$ for all $x, y \in F(T)$.

Example 3.10. Let $X=\mathbb{R}$ with the $b$-metric $d$ given by $d(x, y)=(x-y)^{2}$ for all $x, y \in X$, let $A=[-1,0]$ and $\mathrm{B}=[0,1]$. Define the mapping $\mathrm{T}: \mathrm{A} \cup \mathrm{B} \rightarrow \mathrm{A} \cup \mathrm{B}$ by

$$
\mathrm{T} x=-\frac{x}{8(2+x)} \text { for all } x \in X
$$

It is easy to see that $T$ is a cyclic mapping. Now we will show that the inequality (3.17) holds with $\psi$ and $\varphi$ which are defined by $\psi(t)=k t$ and $\varphi(t)=\frac{k t}{2}$ for all $t \in[0, \infty)$, where $k$ is a fixed nonnegative real number. Let $x \in A$ and $y \in B$. Then we get

$$
\begin{aligned}
\psi\left(2^{3} d(T x, T y)\right) & =8 k\left(\frac{x}{8(2+x)}-\frac{y}{8(2+y)}\right)^{2} \\
& =\frac{k}{2}\left(\frac{x-y}{(2+x)(2+y)}\right)^{2} \leqslant \frac{k}{2}(x-y)^{2}=k(x-y)^{2}-\frac{k}{2}(x-y)^{2}=\psi(d(x, y))-\varphi(d(x, y)) .
\end{aligned}
$$

Therefore, all conditions in Theorem 3.9 are satisfied and so we obtain that $\mathrm{T}$ has a unique fixed point in $A \cap B$, that is, a point $0 \in A \cap B$.

\section{Multidimensional fixed point theorems for $(\alpha, \beta)-(\psi, \varphi)$-contraction mappings type $S$}

In this section, we apply Theorem 3.5 for proving the multidimensional fixed point results. First we shall give some results which are very important for our consideration.

Lemma 4.1. Let $(X, d)$ be a b-metric space with the coefficient $s \geqslant 1$. Define a mapping $D_{n}: X^{n} \times X^{n} \rightarrow \mathbb{R}$ by

$$
D_{n}(A, B)=\max _{1 \leqslant i \leqslant n} d\left(a_{i}, b_{i}\right)
$$

for all $\mathrm{A}=\left(\mathrm{a}_{1}, \mathrm{a}_{2}, \ldots, \mathrm{a}_{\mathrm{n}}\right)$ and $\mathrm{B}=\left(\mathrm{b}_{1}, \mathrm{~b}_{2}, \ldots, \mathrm{b}_{\mathrm{n}}\right)$. Then $\mathrm{D}_{\mathrm{n}}$ is a b-metric on $\mathrm{X}^{\mathrm{n}}$. Moreover, if $(\mathrm{X}, \mathrm{d})$ is complete, then $\left(\mathrm{X}^{\mathrm{n}}, \mathrm{D}_{\mathrm{n}}\right)$ is also complete. 
Lemma 4.2. Let $(X, d)$ be a b-metric space with the coefficient $\mathrm{s} \geqslant 1, \mathrm{~F}: \mathrm{X}^{\mathrm{n}} \rightarrow \mathrm{X}$ be a mapping, and $\{\mathrm{A}, \mathrm{B}\}$ is a partition of $\{1,2, \ldots, n\}$. Suppose that $\Upsilon=\left(\sigma_{1}, \sigma_{2}, \ldots, \sigma_{\mathfrak{n}}\right)$ is an $n$-tuple of mappings from $\{1,2, \ldots, n\}$ into itself verifying $\sigma_{i} \in \Omega_{A, B}$ if $i \in A$ and $\sigma_{i} \in \Omega_{A, B}^{\prime}$ if $i \in B$. If $F_{\curlyvee}: X^{n} \rightarrow X^{n}$ is defined by

$$
F_{\Upsilon}(x)=\left(\begin{array}{l}
F\left(x_{\sigma_{1}(1)}, x_{\sigma_{1}(2)}, \ldots, x_{\sigma_{1}(n)}\right), \\
F\left(x_{\sigma_{2}(1)}, x_{\sigma_{2}(2)}, \ldots, x_{\sigma_{2}(n)}\right), \\
\vdots \\
F\left(x_{\sigma_{n}(1)}, x_{\sigma_{n}(2)}, \ldots, x_{\sigma_{\mathfrak{n}}(n)}\right),
\end{array}\right)
$$

for all $\mathrm{x}=\left(\mathrm{x}_{1}, \mathrm{x}_{2}, \ldots, \mathrm{x}_{\mathrm{n}}\right) \in \mathrm{X}^{\mathrm{n}}$, then the following assertions hold:

1. if $\mathrm{F}$ is continuous, then $\mathrm{F}_{\Upsilon}$ is continuous;

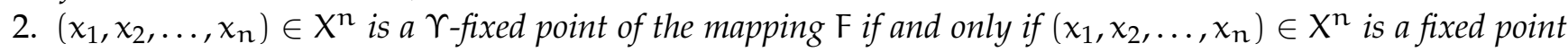
$\mathrm{F} r$.

Theorem 4.3. Let $(\mathrm{X}, \mathrm{d})$ be a complete b-metric space with the coefficient $\mathrm{s} \geqslant 1$, and $\{\mathrm{A}, \mathrm{B}\}$ is a partition of $\{1,2, \ldots, n\}$ and $\Upsilon=\left(\sigma_{1}, \sigma_{2}, \ldots, \sigma_{n}\right)$ be an $n$-tuple of permutations from $\{1,2, \ldots, n\}$ into itself verifying $\sigma_{i} \in$ $\Omega_{\mathrm{A}, \mathrm{B}}$ if $\mathrm{i} \in \mathrm{A}$ and $\sigma_{\mathrm{i}} \in \Omega_{\mathrm{A}, \mathrm{B}}^{\prime}$ if $\mathrm{i} \in \mathrm{B}$. Suppose that $\mathrm{F}: \mathrm{X}^{\mathrm{n}} \rightarrow \mathrm{X}$ and $\mathrm{g}: \mathrm{X} \rightarrow \mathrm{X}$ are two mappings such that there exist $\psi, \varphi \in \Psi$ satisfying

$$
\psi\left(s^{3} d\left(F\left(x_{1}, x_{2}, \ldots, x_{n}\right), F\left(y_{1}, y_{2}, \ldots, y_{n}\right)\right)\right) \leqslant \psi\left(\max _{1 \leqslant i \leqslant n} d\left(x_{i}, y_{i}\right)\right)-\varphi\left(\max _{1 \leqslant i \leqslant n} d\left(x_{i}, y_{i}\right)\right)
$$

for all $\mathrm{x}=\left(\mathrm{x}_{1}, \mathrm{x}_{2}, \ldots, \mathrm{x}_{\mathrm{n}}\right), \mathrm{y}=\left(\mathrm{y}_{1}, \mathrm{y}_{2}, \ldots, \mathrm{y}_{\mathrm{n}}\right) \in \mathrm{X}^{\mathrm{n}}$. If $\mathrm{F}$ is continuous, then $\mathrm{F}$ has a $\Upsilon$-fixed point.

Proof. Let $Y=X^{n}$, then $\left(Y, D_{n}\right)$ is complete b-metric spaces and $F_{\Upsilon}: X^{n} \rightarrow X^{n}$ be define as Lemma 4.2. Since, $F$ is continuous, we have that $F_{\Upsilon}$ is continuous. Let $A=\left(x_{1}, x_{2}, \ldots, x_{n}\right)$ and $B=\left(y_{1}, y_{2}, \ldots, y_{n}\right)$ are element $\mathrm{Y}$, then

$$
\begin{aligned}
D_{n}\left(F_{\curlyvee} A, F_{\curlyvee} B\right) & =D_{n}\left(F_{\curlyvee}\left(x_{1}, x_{2}, \ldots, x_{n}\right), F_{\curlyvee}\left(y_{1}, y_{2}, \ldots, y_{n}\right)\right) \\
& \left.=D_{n}\left(\begin{array}{l}
F\left(x_{\sigma_{1}(1)}, x_{\sigma_{1}(2)}, \ldots, x_{\sigma_{1}(n)}\right), \\
F\left(x_{\sigma_{2}(1)}, x_{\sigma_{2}(2)}, \ldots, x_{\sigma_{2}(n)}\right), \\
\vdots \\
F\left(x_{\sigma_{\mathfrak{n}}(1)}, x_{\sigma_{\mathfrak{n}}(2)}, \ldots, x_{\sigma_{\mathfrak{n}}(n)}\right),
\end{array}\right),\left(\begin{array}{l}
F\left(y_{\sigma_{1}(1)}, y_{\sigma_{1}(2)}, \ldots, y_{\sigma_{1}(n)}\right), \\
F\left(y_{\sigma_{2}(1)}, y_{\sigma_{2}(2)}, \ldots, y_{\sigma_{2}(n)}\right), \\
\vdots \\
\left.F\left(y_{\sigma_{\mathfrak{n}}(1)}\right), y_{\sigma_{\mathfrak{n}}(2)}, \ldots, y_{\sigma_{\mathfrak{n}}(\mathfrak{n})}\right),
\end{array}\right)\right) \\
& =\max _{1 \leqslant i \leqslant n} d\left(F\left(x_{\sigma_{\mathfrak{i}}(1)}\right), F\left(x_{\sigma_{\mathfrak{i}}(2)}\right), \ldots, F\left(x_{\sigma_{\mathfrak{i}}(\mathfrak{n})}\right), F\left(y_{\sigma_{\mathfrak{i}}(1)}\right), F\left(y_{\sigma_{\mathfrak{i}}(2)}\right), \ldots, F\left(y_{\sigma_{\mathfrak{i}}(\mathfrak{n})}\right)\right) .
\end{aligned}
$$

Hence,

$$
\begin{aligned}
\psi\left(s^{3} D_{n}\left(F_{\curlyvee} A, F_{\curlyvee} B\right)\right) & =\psi\left(s^{3} \max _{1 \leqslant i \leqslant n} d\left(F\left(x_{\sigma_{i}(1)}, x_{\sigma_{i}(2)}, \ldots, x_{\sigma_{i}(n)}\right), F\left(y_{\sigma_{i}(1)}, y_{\sigma_{i}(2)}, \ldots, y_{\sigma_{i}(n)}\right)\right)\right. \\
& \leqslant \psi\left(\max _{1 \leqslant i \leqslant n} d\left(x_{i}, y_{i}\right)\right)-\varphi\left(\max _{1 \leqslant i \leqslant n} d\left(x_{i}, y_{i}\right)\right)=\psi\left(D_{n}(A, B)\right)-\varphi\left(D_{n}(A, B)\right) .
\end{aligned}
$$

Setting $\alpha, \beta: Y \rightarrow[0, \infty)$ by $\alpha_{\mathfrak{n}}(A)=s, \beta_{\mathfrak{n}}(B)=s$ for all $A, B \in Y$, and using Theorem 3.5, we get $F_{\curlyvee}$ has a fixed point and thus $F$ has a $\Upsilon$-fixed point.

\section{An application to nonlinear integral equation}

In this section, we give an applications of our main result for showing the existence theorem of a solution for the following nonlinear integral equation:

$$
x(t)=\phi(t)+\lambda \int_{a}^{b} h(t, s) f(s, x(s)) d s,
$$

where $\lambda \in[0, \infty)$ and $a, b \in \mathbb{R}$ such that $a<b, x \in C[a, b]$ (the set of all continuous functions from $[a, b]$ into $\mathbb{R}), \phi:[a, b] \rightarrow \mathbb{R}, h:[a, b] \times[a, b] \rightarrow[0, \infty)$ and $f:[a, b] \times \mathbb{R} \rightarrow \mathbb{R}$ are given continuous mappings. 
Theorem 5.1. Consider the nonlinear integral equation (5.1). Suppose that the following conditions hold:

(A) $f:[a, b] \times \mathbb{R} \rightarrow \mathbb{R}$ is nondecreasing in the second ordered with $f(t, r) \geqslant 0$ for all $t \in[a, b]$ and $r \in \mathbb{R}$;

(B) there exists a constant $p \geqslant 1$ such that

$$
\sup _{t \in[a, b]}\left(\int_{a}^{b} h(t, s)^{p} d s\right)<\frac{1}{2^{3 p^{2}-3 p} \lambda^{p}(b-a)^{p-1} L^{p}} ;
$$

(C) there exist $\theta, \vartheta: C[a, b] \rightarrow \mathbb{R}$ that satisfy the following condition:

$\left(\mathrm{C}_{1}\right)$ for each $\mathrm{x}, \mathrm{y} \in \mathrm{C}[\mathrm{a}, \mathrm{b}]$ with $\theta(\mathrm{x}) \geqslant 0$ and $\vartheta(\mathrm{y}) \geqslant 0$, we have

$$
|f(t, x(r))-f(t, y(r))| \leqslant L\left[\zeta\left((x(r)-y(r))^{p}\right)\right],
$$

where $\mathrm{L} \geqslant 0$ and $\zeta:[0, \infty) \rightarrow[0, \infty)$ is a right continuous nondecreasing function such that $\zeta(0)=0$ $\zeta(\mathrm{t})<\mathrm{t}$ for all $\mathrm{t}>0$;

$\left(C_{3}\right)$ there exists $x_{\diamond} \in C[a, b]$ such that $\theta\left(x_{\diamond}\right) \geqslant 0$ and $\vartheta\left(x_{\diamond}\right) \geqslant 0$;

$\left(\mathrm{C}_{4}\right)$ if

$$
\theta(x) \geqslant 0 \text { for some } x \in C[a, b] \text { implies } \vartheta(T x) \geqslant 0
$$

and

$$
\vartheta(\mathrm{x}) \geqslant 0 \text { for some } \mathrm{x} \in \mathrm{C}[\mathrm{a}, \mathrm{b}] \text { implies } \theta(\mathrm{T} x) \geqslant 0,
$$

where $\mathrm{F}: \mathrm{C}[\mathrm{a}, \mathrm{b}] \rightarrow \mathrm{C}[\mathrm{a}, \mathrm{b}]$ defined by

$$
(T x)(t)=\phi(t)+\lambda \int_{a}^{b} h(t, s) f(s, x(s)) d s
$$

for all $\mathrm{x} \in \mathrm{C}[\mathrm{a}, \mathrm{b}]$ and $\mathrm{t} \in[\mathrm{a}, \mathrm{b}]$;

$\left(C_{5}\right)$ if $\left\{x_{n}\right\}$ is a sequence in $C[a, b]$ that converges to $x \in C[a, b]$ and $\theta\left(x_{n}\right) \geqslant 0$ for all $n \in \mathbb{N}$, then $\vartheta(x) \geqslant 0$.

Then the nonlinear integral equation (5.1) has a solution.

Proof. Let $\mathrm{X}=\mathrm{C}[\mathrm{a}, \mathrm{b}]$ and $\mathrm{d}: \mathrm{X} \times \mathrm{X} \rightarrow[0, \infty)$ be defined by

$$
d(x, y)=\sup _{t \in[a, b]}|x(t)-y(t)|^{p}
$$

for all $x, y \in X$. Then $(X, d)$ is a complete $b$-metric space with $s=2^{p-1}$. Also, we define $\alpha, \beta: X \rightarrow[0, \infty)$ by

$$
\alpha(x)=\left\{\begin{array}{ll}
2^{p-1}, & \text { if } \theta(x) \geqslant 0, \\
0, & \text { otherwise, }
\end{array} \quad \text { and } \quad \beta(x)= \begin{cases}2^{p-1}, & \text { if } \vartheta(x) \geqslant 0, \\
0, & \text { otherwise. }\end{cases}\right.
$$

Here we will show that $T$ is an $(\alpha, \beta)-(\psi, \varphi)$-contraction mapping type $S$. Suppose that $x, y \in X$ such that $\alpha(x) \beta(y) \geqslant s$ and then $\theta(x) \geqslant 0$ and $\vartheta(y) \geqslant 0$. Putting $q:=\frac{p}{p-1} \in \mathbb{R}$, that is, $\frac{1}{p}+\frac{1}{q}=1$, by condition $\left(C_{1}\right)$ and the Hölder inequality, for each $t \in[a, b]$ we get

$$
\begin{aligned}
\left(2^{3 p-3}|(T x)(t)-(T y)(t)|\right)^{p} & =2^{3 p^{2}-3 p}\left|\left(\phi(t)+\lambda \int_{a}^{b} h(t, s) f(s, x(s)) d s\right)-\left(\phi(t)+\lambda \int_{a}^{b} h(t, s) f(s, y(s)) d s\right)\right|^{p} \\
& \leqslant 2^{3 p^{2}-3 p} \lambda^{p}\left(\int_{a}^{b} h(t, s)|f(s, x(s))-f(s, y(s))| d s\right)^{p} \\
& \leqslant 2^{3 p^{2}-3 p} \lambda^{p}\left(\left(\int_{a}^{b} 1^{q}\right)^{1 / q}\left[\int_{a}^{b} h(t, s)^{p}|f(s, x(s))-f(s, y(s))|^{p} d s\right]^{1 / p}\right)^{p} \\
& \leqslant 2^{3 p^{2}-3 p} \lambda^{p}(b-a)^{p / q}\left[\int_{a}^{b} h(t, s)^{p}\left(L\left[\zeta\left((x(s)-y(s))^{p}\right]\right)^{p} d s\right)\right]
\end{aligned}
$$




$$
\begin{aligned}
& \left.\leqslant 2^{3 p^{2}-3 p} \lambda^{p}(b-a)^{p / q}\left[\int_{a}^{b} h(t, s)^{p}\left(L^{p}\left[\zeta\left(\sup _{t \in[a, b]}|x(s)-y(s)|^{p}\right)\right]^{p} d s\right)\right]\right] \\
& \leqslant 2^{3 p^{2}-3 p} \lambda^{p}(b-a)^{p-1}\left(\int_{a}^{b} h(t, s)^{p} d s\right) L^{p} \zeta\left(\sup _{t \in[a, b]}|x(s)-y(s)|^{p}\right)^{p} \\
& <[\zeta(d(x, y))]^{p} .
\end{aligned}
$$

Now, we define functions $\psi, \varphi:[0, \infty) \rightarrow[0, \infty)$ by $\psi(t)=t^{p}$ and $\varphi(t)=t^{p}-(\zeta(t))^{p}$ for all $t \in[0, \infty)$. Hence, from (5.2), we have

$$
\psi\left(s^{3}(d(T x, T y)) \leqslant(d(T x, T y))^{p}-\left[(d(T x, T y))^{p}-(\zeta(d(T x, T y)))^{p}\right] .\right.
$$

Therefore, $T$ is an $(\alpha, \beta)-(\psi, \varphi)$-contraction mapping type $S$.

It is easy to see that the other conditions of Theorem 3.5 hold and hence all conditions of Theorem 3.5 are satisfied. Consequently, we obtain that $\mathrm{T}$ has a fixed point, that is, the nonlinear integral equation (5.1) has a solution.

\section{Acknowledgment}

The author would like to thank the Kasetsart University Research and Development Institute (KURDI) for financial support. The second author would like to thank the Thailand Research Fund and Thammasat University under Grant No. MRG5980242 for financial support during the preparation of this manuscript.

\section{References}

[1] M. Abbas, D. Dorić, Common fixed point theorem for four mappings satisfying generalized weak contractive condition, Filomat, 24 (2010), 1-10. 2.1

[2] A. Aghajani, M. Abbas, J. R. Roshan, Common fixed point of generalized weak contractive mappings in partially ordered b-metric spaces, Math. Slovaca, 64 (2014), 941-960. 2.19

[3] Y. I. Alber, S. Guerre-Delabriere, Principle of weakly contractive maps in Hilbert spaces, in: New Results in Operator Theory and its Applications, Theory Adv. Appl., 98 (1997), 7-22. 1, 2.1

[4] S. Alizadeh., F. Moradloub, P. Salimic, Some Fixed Point Results for $(\alpha, \beta)-(\psi, \phi)$-Contractive Mappings, Filomat, 28 (2014), 635-647. 1, 2.2, 2.11, 2.12

[5] A. D. Arvanitakis, A proof of the generalized Banach contraction conjecture, Proc. Amer. Math. Soc., 131 (2003), 36473656. 1

[6] S. Banach, Sur les opérations dans les ensembles abstraits et leurs applications aux équations intégrales, Fund. Math., 3 (1922), 133-181. 2.1, 2.1

[7] V. Berinde, Approximating fixed points of weak contractions using the Picard iteration, Nonlinear Anal. Forum, 9 (2004) 43-53. 1

[8] M. Boriceanu, M. Bota, A. Petrusel, Multivalued fractals in b-metric spaces, Cent. Eur. J. Math., 8 (2010), $367-377$. $2.18,2.20,2.21,2.22$

[9] L. B. C̀iric̀, A generalization of Banach principle, Proc. Amer. Math. Soc., 45 (1974), 267-273. 1

[10] S. Czerwik, Contraction mappings in b-metric spaces, Acta Math. Inform. Univ. Ostraviensis, 1 (1993), 5-11. 2.3, 2.15

[11] D. D. Dorić, Common fixed point for generalized ( $\psi, \phi)$-weak contractions, Appl. Math. Lett., 22 (2009), 1896-1900. 2.1, 2.8

[12] P. N. Dutta, B. S. Choudhury, A generalisation of contraction principle in metric spaces, Fixed Point Theory Appl., 2008 (2008), 8 pages. 1, 2.1

[13] M. A. Geraghty, On contractive mappings, Proc. Amer. Math. Soc., 40 (1973), 604-608. 1

[14] M. S. Khan, M. Swaleh, S. Sessa, Fixed points theorems by altering distances between the points, Bull. Aust. Math. Soc., 30 (1984), 1-9. 1, 2.1, 2.5

[15] B. E. Rhoades, A comparison of various definitions of contractive mappings, Trans. Amer. Math. Soc., 226 (1977), $257-90$. 1

[16] B. E. Rhoades, Some theorems on weakly contractive maps, Nonlinear Anal., 47 (2001), 2683-2693. 1, 2.1, 2.2, 2.1, 2.4

[17] A. Roldán, J. Martínez-Moreno, C. Roldán, Multidimensional fixed point theorems in partially ordered complete metric spaces, J. Math. Anal. Appl., 396 (2012), 536-545. 2.23

[18] B. Samet, C. Vetro, P. Vetro, Fixed point theorems for $\alpha-\psi$-contractive type mappings, Nonlinear Anal., 4 (2012), 2154-2165. 1, 2.2, 2.9, 2.10

[19] W. Sintunavarat, Nonlinear integral equations with new admissibility types in b-metric spaces, J. Fixed Point Theory Appl., 18 (2016) 397-416. 1, 2.2, 2.13, 2.14, 2.17 